\title{
Multiplexed FBG Monitoring System for Forecasting Coalmine Water Inrush Disaster
}

\author{
B. Liu, ${ }^{1}$ S. C. Li, ${ }^{1}$ J. Wang, ${ }^{2,3}$ Q. M. Sui, ${ }^{2}$ L. C. Nie, ${ }^{1}$ and Z. F. Wang ${ }^{2}$ \\ ${ }^{1}$ Geotechnical \& Structural Engineering Research Center, Shandong University, Shandong, Jinan 250061, China \\ ${ }^{2}$ Optical Fiber Sensing Technology and Engineering Research Center, Shandong University, Shandong, Jinan 250061, China \\ ${ }^{3}$ School of Earth Science and Engineering, Nanjing University, Jiangsu, Nanjing 210093, China
}

Correspondence should be addressed to B. Liu, 36682145@qq.com and J. Wang, wangjing329@mail.sdu.edu.cn

Received 7 August 2012; Revised 11 October 2012; Accepted 8 November 2012

Academic Editor: Jayanta K. Sahu

Copyright (C) 2012 B. Liu et al. This is an open access article distributed under the Creative Commons Attribution License, which permits unrestricted use, distribution, and reproduction in any medium, provided the original work is properly cited.

This paper presents a novel fiber-Bragg-grating- (FBG-) based system which can monitor and analyze multiple parameters such as temperature, strain, displacement, and seepage pressure simultaneously for forecasting coalmine water inrush disaster. The sensors have minimum perturbation on the strain field. And the seepage pressure sensors adopt a drawbar structure and employ a corrugated diaphragm to transmit seepage pressure to the axial strain of FBG. The pressure sensitivity is $20.20 \mathrm{pm} / \mathrm{KPa}$, which is $6 \mathrm{E} 3$ times higher than that of ordinary bare FBG. The FBG sensors are all preembedded on the roof of mining area in coalmine water inrush model test. Then FBG sensing network is set up applying wavelength-division multiplexing (WDM) technology. The experiment is carried out by twelve steps, while the system acquires temperature, strain, displacement, and seepage pressure signals in real time. The results show that strain, displacement, and seepage pressure monitored by the system change significantly before water inrush occurs, and the strain changes firstly. Through signal fusion analyzed it can be concluded that the system provides a novel way to forecast water inrush disaster successfully.

\section{Introduction}

As is known, FBG sensors are light weight, small size, easy to install, durable, corrosion resistant, water-proof, immune to electromagnetic interference, and so forth [13]. Furthermore, FBG sensors can be used to monitor large structures thanks to the inherent capability of multiplexing many sensors, which allows the measurement of several parameters at different places. In addition, FBG system can be employed as real-time monitor. Because of these intrinsic natures, FBG sensors are excellent for measuring static and dynamic measurement, such as temperature, strain, displacement, and pressure [4-9]. For FBG pressure sensors, pressure sensitivity is the main restriction. In [10] a novel FBG pressure sensor structure is proposed, which is based on a carbon fiber ribbon-wound composite cylindrical shell (CFRCCS). The sensor has a moderate measurement range $(8 \mathrm{MPa})$ and high sensitivity $(0.45 \mathrm{~nm} / \mathrm{MPa})$. Since FBG strain sensors are mostly encapsulated by stainless steel and other materials, they have an impact on the strain field of the surrounding rock or soil. Meanwhile, there are several kinds of FBG displacement sensors used in engineering, yet they are large in volume and imprecise. Therefore, continuing works on fiber sensors encapsulation are requisite.

Meanwhile, FBG-based sensing systems have been employed in geotechnical engineering and model test tentatively. As early as in 1994, Ferdinand et al. reported on the European BRITE/EURAM STABILOS Project, whose main purpose was to produce a new geotechnical instrument based on optical fiber sensing technology applied to measurements of load and displacement changes in underground excavations of mines, tunnels, or storage caverns. In the project, FBG strain sensors and FBG temperature sensors were designed. Although at the time FBG fabricating technology and fiber sensing technology were immature, it had important reference value to the present study [11]. Currently, Kerrouche et al. employed FBG strain sensors and FBG temperature sensors to perform structural integrity monitoring of the Mjosund bridge in western Norway, [12], and $\mathrm{Li}$ et al. used 10 metal groove encapsulated sensors 
to measure the surface strain of second lining of a tunnel [13]. The results demonstrated that FBG sensors could operate successfully in a continuous manner and under harsh environmental conditions. Zhu et al. and Li et al. designed quasi-distributed FBG displacement sensors mounted on a slender bar to monitor deformation of a dam test model $[14,15]$. Although the above results are all satisfactory, FBG sensors are still simple in encapsulation and low in sensitivity, and they cannot be used in restricted spaces or on diminutive structures. In addition, the sensing network is simple and detecting parameter is unitary.

However, underground mining activities will inevitably result in the redistribution of rock stress and the damage of rock, which might greatly change the permeability of the rock and induce possible water inrush at the roof [16]. Therefore, the process of water inrush has prewarning information such as the variation of temperature, strain, displacement, and permeability in the surrounding rock. The aims of our research project are to get the relation of temperature, strain, displacement, and seepage pressure with water inrush to analyze the formation mechanism of water inrush by using a geological model test, and to successfully forecast the impending disaster employing a fiber-optic sensing system. To the best of our knowledge, the systematic research on prewarning regarding information of water inrush using fiber-optic technology has not been studied to date.

In this research, FBG seepage pressure sensors based on draw-bar structure and corrugated diaphragm, FBG strain sensors based on similar material blocks, FBG displacement sensors based on spring, and FBG temperature sensors based on dual-pipe structure are all designed and fabricated especially for the coalmine water inrush model test. An integrated multiparameter monitoring system is set up, to monitor the four parameters synchronously and to analyze them on-line. The experimental results are shown here, and the prototype forecasts water inrush about 35 minutes before the collapse of the roof of the mine area.

\section{Development of FBG Sensors}

It is well known that the FBG-based sensor is wavelengthcoded, and the corresponding Bragg wavelength of a FBG is defined by

$$
\lambda_{B}=2 n_{\mathrm{eff}} \Lambda
$$

where $n_{\text {eff }}$ is the effective refractive index of the optical fiber, and $\Lambda$ is the grating period.

The shift of the reflected Bragg wavelength represented in the linear relationship is shown in (2):

$$
\Delta \lambda_{B}=\lambda_{B}\left[(\alpha+\xi) \Delta T+\left(1-p_{e}\right) \varepsilon\right],
$$

where $\varepsilon$ is the strain, $\Delta T$ is the variation of temperature, $\alpha$ is the fiber linear thermal coefficient, $\xi$ is the thermooptic coefficient, and $p_{e}$ is the effective photo-elastic coefficient.

In this paper, FBGs were written, in-house, using a phase mask technique at nominal wavelengths of $1510 \mathrm{~nm} \sim$ $1590 \mathrm{~nm}$ with characteristics of $10 \mathrm{~mm}$ length, a reflectivity of more than $90 \%$, and bandwidths of $0.2 \mathrm{~nm}$ typically.

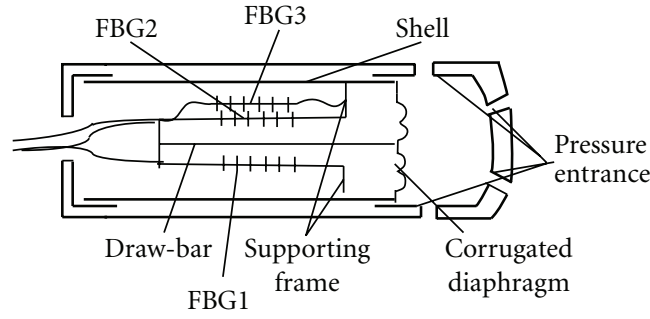

FIGURE 1: Sketch of FBG seepage pressure sensor.

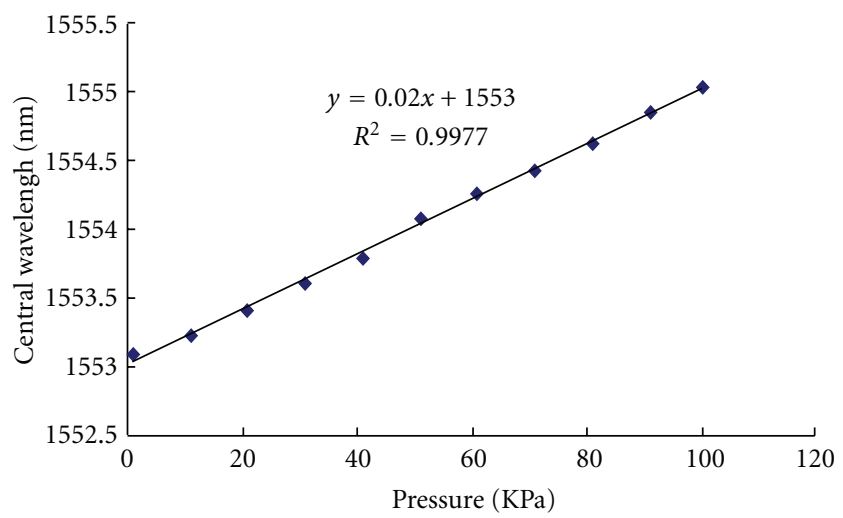

FIGURE 2: Calibration curve of FBG seepage pressure sensor.

2.1. FBG Seepage Pressure Sensor. FBG seepage pressure sensor has been designed based on corrugated diaphragm and draw-bar structure. It mainly consists of shell, draw-bar, corrugated diaphragm, support frame, and FBG, as shown in Figure 1. One end of FBG1 and FBG2 which are used to detect seepage pressure is fixed on draw-bar, and the other one is fixed on support frame. They are straight, while FBG 3 which is used to detect temperature is crooked in free-state.

Corrugated diaphragm is pressure-sensitive device of FBG seepage pressure sensor, which determines the sensitivity and testing range of the sensor. The principle of corrugated diaphragm is shown in [17]. Central displacement of corrugated diaphragm is

$$
w_{0}=\frac{p R}{E}\left(\frac{R}{h}\right)^{3} \frac{\bar{w}}{\bar{p}},
$$

where $p$ is the pressure on diaphragm, in $\mathrm{MPa} ; R$ is working radius, in $\mathrm{mm}$; $h$ is thickness, in $\mathrm{mm}$; $E$ is Young's modulus, in $\mathrm{MPa} ; \bar{w} / \bar{p}$ is dimensionless parameter and it has relation with depth of wave and thickness of diaphragm.

Assuming that the length of fiber under draught is $L$ and strain sensitivity coefficient is $K_{e}$, variation of wavelength $\Delta \lambda_{B}=K_{e} \varepsilon \lambda_{B}, \varepsilon=w_{0} / L$, (4) will be induced from (3). In (4), variation of wavelength $\Delta \lambda_{B}$ is directly proportional to the pressure $p$ :

$$
\Delta \lambda_{B}=K_{e} \frac{w_{o}}{L} \lambda_{B}=K_{e} \frac{p R}{L E}\left(\frac{R}{h}\right)^{3} \frac{\bar{w}}{\bar{p}} \lambda_{B} .
$$

In this paper, the material of the corrugated diaphragm is elastic alloy Ni36CrTiAl (3J1) and the Young's modulus E is 


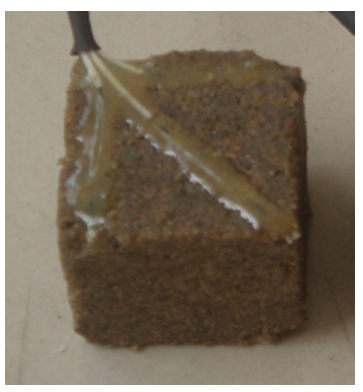

(a) Fiber grating strain block

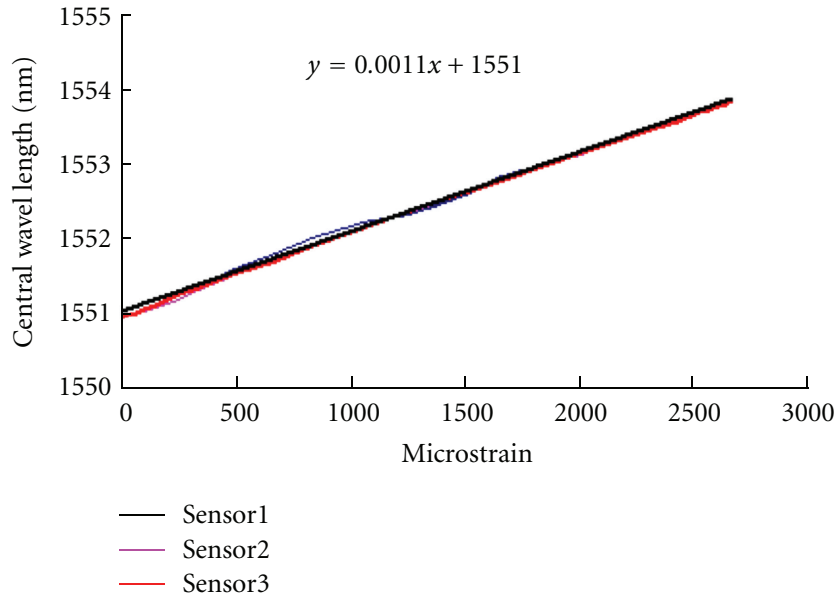

(b) Sensing characteristics

Figure 3: Fiber grating strain sensor.

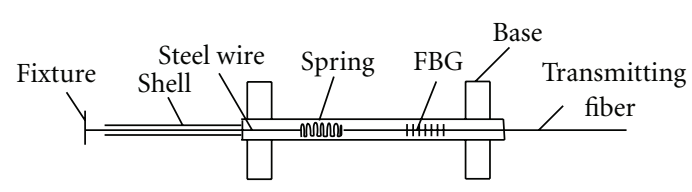

(a) Sketch of FBG displacement sensor

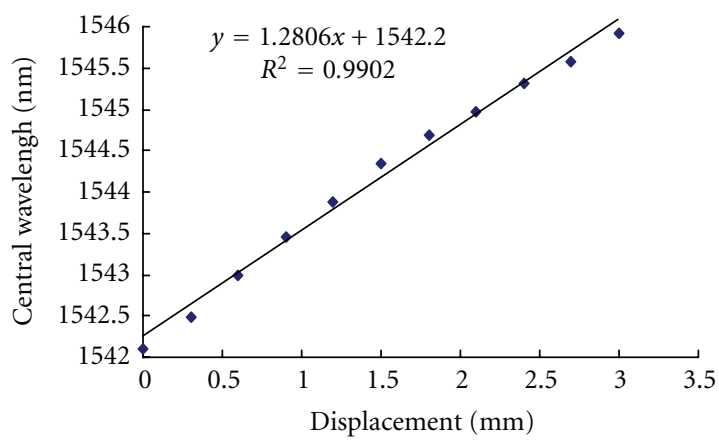

(b) Calibration curve of FBG displacement sensor

Figure 4: FBG displacement sensor.

$210 \mathrm{GPa}$. The thickness $h$ of the diaphragm is $0.1 \mathrm{~mm}$ and the working radius $R$ is $8.0 \mathrm{~mm}$. As the corrugations along the radius have a constant depth, the value of the dimensionless parameter $\bar{p} / \bar{w}=36$.

The principle of the FBG seepage pressure sensor is as follows: the central point of the corrugated diaphragm moves under pressure, and the draw-bar moves simultaneously, which gives a pull to FBG 1 and the central wavelength of FBG changes eventually. When the corrugated diaphragm is under the pressure of $100 \mathrm{KPa}$, the central displacement is $0.068 \mathrm{~mm}$. assuming that strain coefficient of optical fiber is the same as that of FBG, the strain of FBG1 and FBG2 is $2125 \mu \varepsilon$ when the pressure is $100 \mathrm{KPa}$. As strain coefficient of FBG1 is $1.2 \mathrm{pm} / \mu \varepsilon$ [18], then the theoretical value of the central wavelength's variation is $2.55 \mathrm{~nm}$. Moreover, finite element simulation of the seepage pressure sensor has been done, and the result is $2.53 \mathrm{~nm}$, which is detailed in [19].

Pressure-sensitive calibration experiment is carried out under constant temperature condition. Pressure increases gradually. For a final pressure of $100 \mathrm{KPa}$, the curve of central wavelength as a function of pressure is shown in Figure 2.

In Figure 2, correlation coefficient is close to 1.0, and pressure sensitivity is $20.0 \mathrm{pm} / \mathrm{KPa}$, while pressure sensitivity of ordinary FBG is only $-3.0 E-3 \mathrm{pm} / \mathrm{KPa}$ [20]. As a result, pressure sensitivity of the senor is $6 \mathrm{E} 3$ times higher than that of ordinary FBG.

In the experiment, the variation of the central wavelength is $1.93 \mathrm{~nm}$, which is smaller than the theoretical value, $2.55 \mathrm{~nm}$ and the simulation value, $2.53 \mathrm{~nm}$. The main reason is that FBG1 gives the draw bar a counterforce which reduces central displacement of the diaphragm while the diagram pulls draw bar.

2.2. FBG Strain Sensors. Since FBG strain sensors widely used are encapsulated employing metal, their Young's modulus is too great to match with the surrounding material. Furthermore, they can not detect shear strain. Then FBG strain blocks designed specially for the coalmine water inrush model test are fabricated to overcome the common shortcomings of stress and strain sensors. They are shown in Figure 3; three fiber Bragg gratings are pasted on one surface of the sensing block to test horizontal strain, vertical strain, and shear strain separately. From (2) it can also be seen that $\Delta \lambda_{B}$ is linear with $\varepsilon$ without the effect of temperature and other quantities. Strain sensitivity of the sensor is $1.1 \mathrm{pm} / \mu \varepsilon$, 


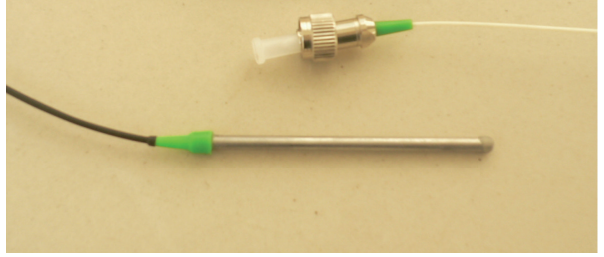

(a) FBG temperature sensor

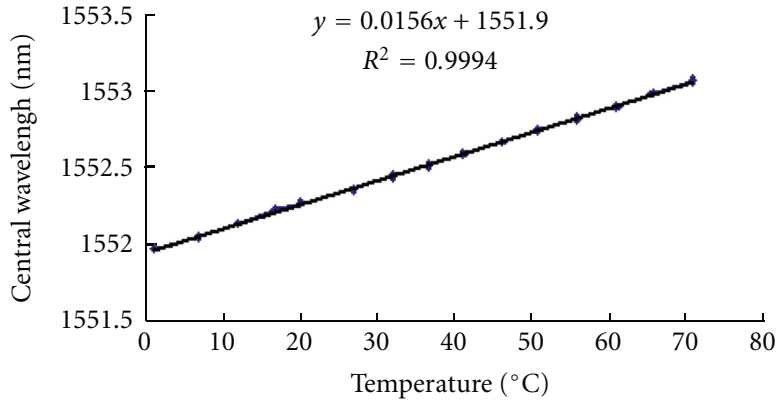

(b) Calibration curve of FBG temperature sensor

FIgURE 5: FBG temperature sensor.

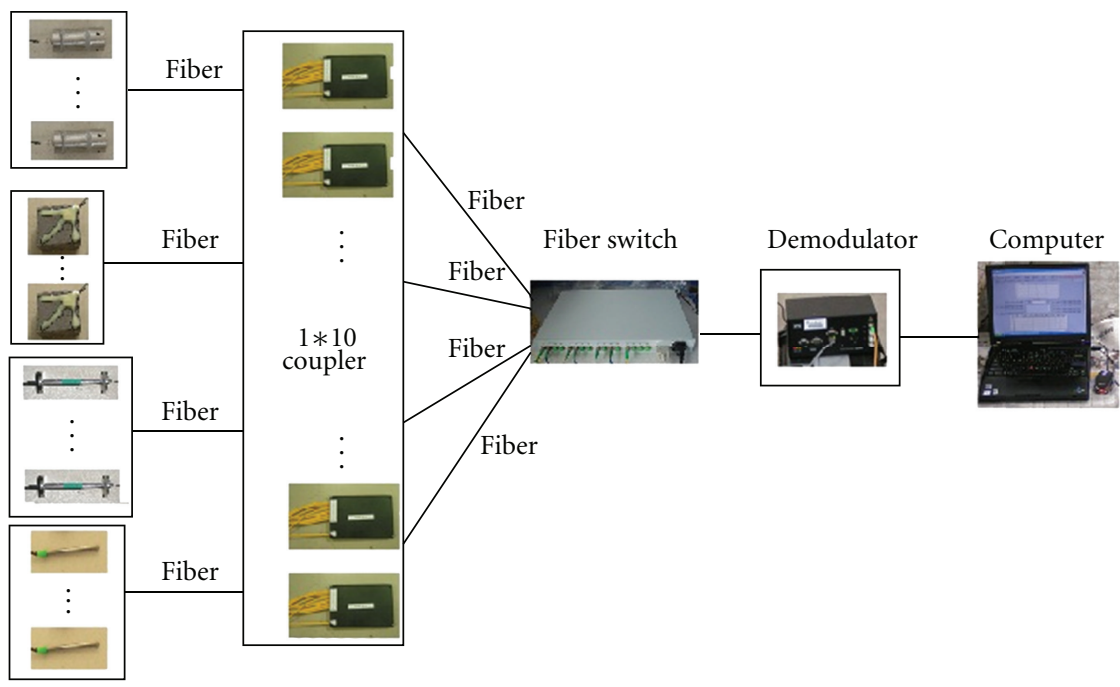

(a) The architecture of the system

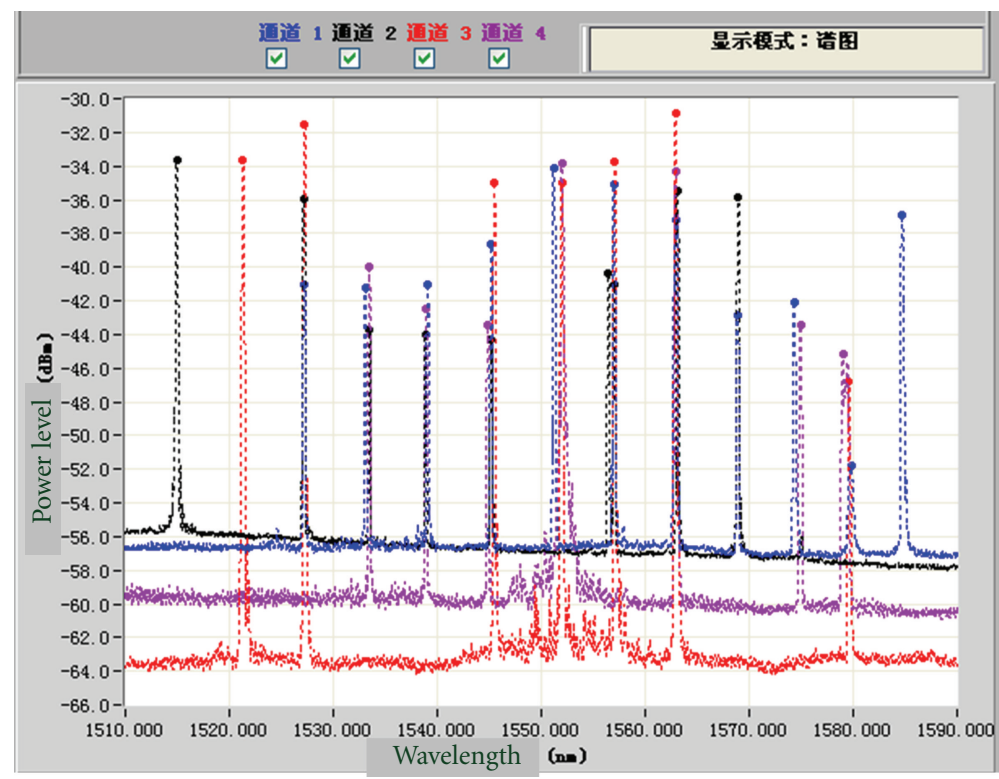

(b) The spectra of the FBG sensors

FIGURE 6: Sketch of the system. 


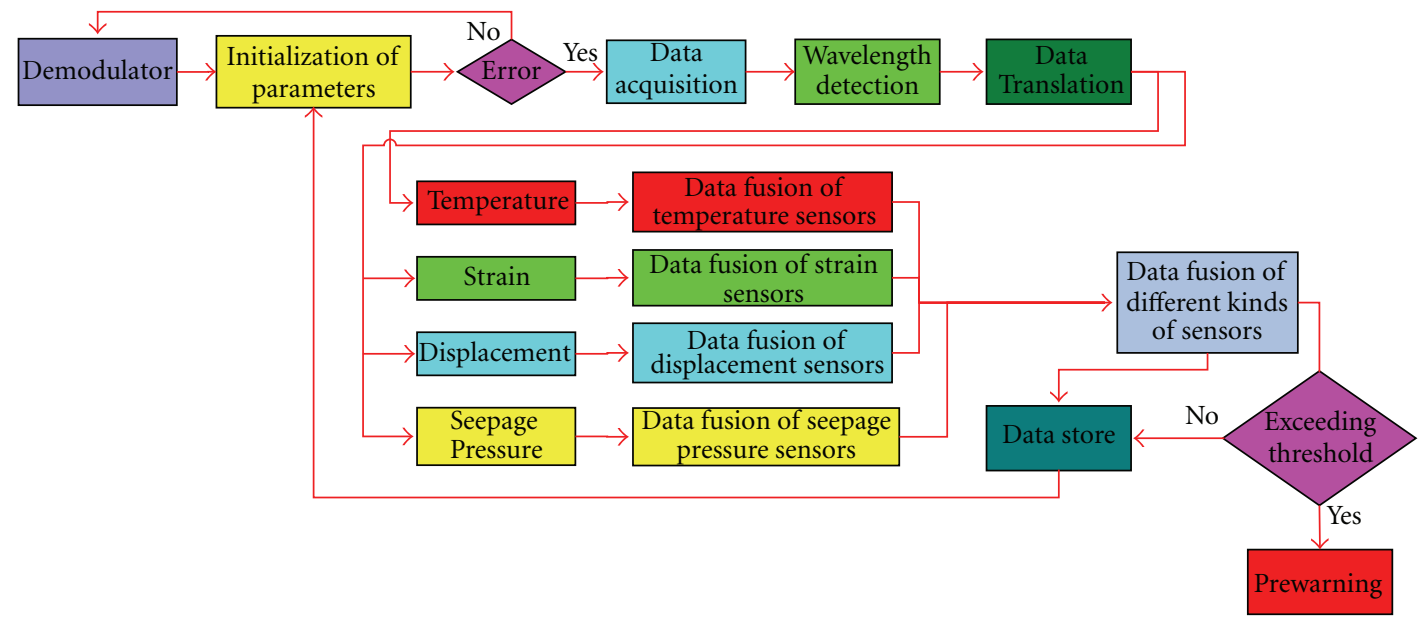

FIgURE 7: Flow chart of the software (not only temperature, strain, displacement, and seepage pressure signal but also the fusion data is beyond safe range, it will forecast the disaster and give the monitoring result automatically).

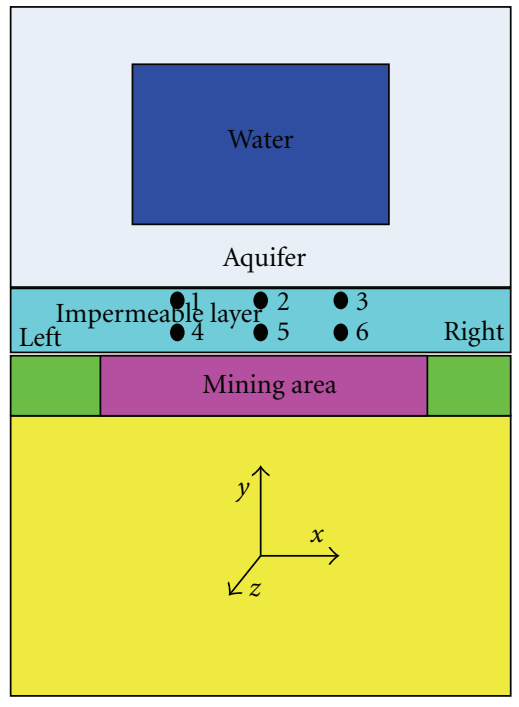

(a)

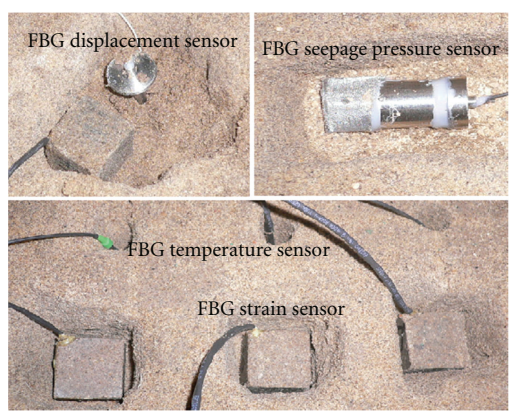

(b)

FIGURE 8: Coalmine water inrush model test, (a) sketch of the water inrush model test, (b) burying process of FBG sensors.

which is smaller than the ordinary value $1.2 \mathrm{pm} / \mu \varepsilon$ [18] because the strain transferring coefficient is $91.7 \%$. And in Figure 3(b), sensor1, sensor2, and sensor3 are used to test horizontal strain, vertical strain, and shear strain separately. They have been used in other model test, and they have more advantages than resistance strain gauge, which are detailed in [21].

\subsection{FBG Displacement Sensor and Temperature Sensor. FBG} displacement sensor as shown in Figure 4 consists of FBG, spring, shell, and the base. Shell of the sensor is stainless steel pipe with the diameter of $5 \mathrm{~mm}$ and the length of $50 \mathrm{~mm}$, while the base of the sensor is stainless steel lamellar structure with the diameter of $20 \mathrm{~mm}$ and the thickness of $2 \mathrm{~mm}$. The fixture is fixed at reference location (assuming it is stationary), and the base is fixed on the testing point. When other parameters are defined, axial elongation or compression of the FBG is directly proportional to the displacement.

A fiber Bragg grating temperature sensor based on dualpipe structure is developed, which is shown in Figure 5. It is only $30 \mathrm{~mm}$ long. Outer cannula and inner cannula of the sensor are both made of stainless steel with the dimension of $\phi 3 \times 30 \mathrm{~mm}$ and $\phi 1 \times 25 \mathrm{~mm}$, respectively. From (2) it can be seen that $\Delta \lambda_{B}$ is linear with $\Delta T$ without effect of strain and other quantities. The temperature sensitivity of the sensor is $15.6 \mathrm{pm} /{ }^{\circ} \mathrm{C}$, and the linearity is 0.99 .

\section{Development of the System}

3.1. The Hardware. As shown in Figure 6(a), the multiplexed FBG monitoring system mainly consists of a computer, a FBG demodulator with four channels, a fiber switch with 


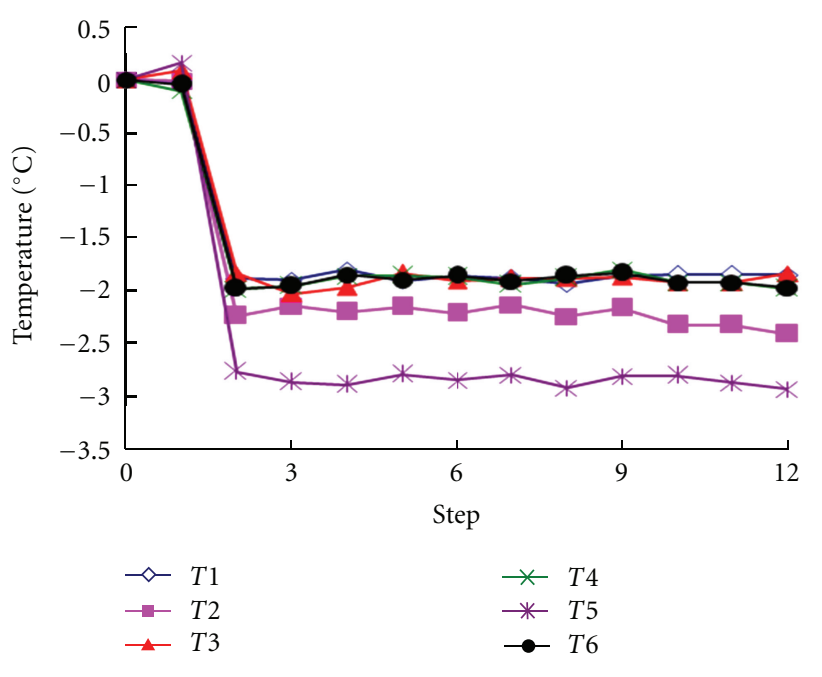

FIGURE 9: Curves of temperature.

eight output channels, thirty three FBGs, two $1 \times 9$ couplers, four $1 \times 3$ couplers, and one $1 \times 2$ coupler. The FBG demodulator transforms optical signal to electrical signal. The wavelength resolution of FBG demodulator which is produced by MOI is $1.0 \mathrm{pm}$, and couplers are produced by Shanghai Hanyu Company. And the spectra of the FBG sensors are shown in Figure 6(b). The wavelengths of FBG sensors in one channel are different from each other, but The FBG sensors in different channels can have the same wavelength. Complex FBG network technology using WDM and SDM technology is adopted which can reduce the cost of the system. Meanwhile, wavelength encoding technology is employed: central wavelength interval of FBG temperature sensors in the first and the second channel is $2.0 \mathrm{~nm}$, while central wavelength interval of strain sensors in the third and the forth channel, displacement sensors in the fifth and the sixth channel, and seepage pressure sensors in the seventh and the eighth channel is $4.0 \mathrm{~nm}$. The sensors in the same channel have different central wavelengths, so that it can be used to monitor spatially distributed parameters. In order to improve the survival rate of the sensor and to increase reliability of the system, all sensors are connected in parallel.

The performance indexes of the system are as follows: temperature range: $-20^{\circ} \mathrm{C} \sim 100^{\circ} \mathrm{C}$, temperature resolution: $0.1^{\circ} \mathrm{C}$, strain range: $-2 E 3 \mu \varepsilon \sim+2 E 3 \mu \varepsilon$, strain resolution: $1 \mu \varepsilon$, displacement range: $-0 \mathrm{~mm} \sim 3 \mathrm{~mm}$, displacement resolution: $1 \mu \varepsilon$, seepage pressure range: $0 \mathrm{KPa} \sim 100 \mathrm{KPa}$, and seepage pressure resolution: $0.5 \mathrm{KPa}$.

3.2. The Software. In the system, the information detected by a single kind of sensors is more or less uncertain. It mainly appear as imperfection, unreliability, and imprecision. Therefore, multiparameter information fusion technology which consists of sensors fusion and final information fusion detected by different kinds of sensors is adopted to improve accuracy of the system. Then neural network technology is used to analyze the information detected by the sensors and forecast the water inrush disaster.
The software mainly consists of a low-level computer procedure, host computer procedure, and procedure interface. The lower computer procedure adopts LabVIEW, while host computer procedure uses $\mathrm{C}++$ Builder. The low-level computer procedure acquires central wavelength signal of different kinds of sensors and stores them to database. While the host computer procedure reads central wavelength signal from database and transforms them to temperature, strain, displacement, and seepage pressure, respectively. Then it analyzes the signals synthetically using signals fusion technology. Furthermore, the software can prewarn about disaster automatically. The flow chart of the software is shown in Figure 7.

Moreover, monitoring results can be transmitted to the remote monitoring room through optical fiber cable, so optical signal and the electric signal are separated in this system (there are only optical signal underground, and photoelectric conversion and signal processing are executed in the control room). These important attributes are necessary for the realization of a practical long-range, real-time monitoring system.

\section{Application in Coalmine Water Inrush Model Test}

4.1. Introduction of Water Inrush Model Test. In order to explore the relation between several parameters and water inrush, to discover the origin of coalmine water inrush, and to apply such knowledge to analyze the probability of disaster in practical engineering, a coalmine water inrush model consisting of mining area, impermeable layer [22], aquifer, and water body was established, which is shown in Figure 8.

Monitoring points were set in the impermeable layer which was between the aquifer and mining area. There was 6 monitoring points arranged in two layers. FBG sensors were embedded in the model. Model-making process was as follows: firstly, the monitoring points were excavated in the solid similar material, and then the bottom of the hole was planed. Secondly, FBG sensors were placed in the corresponding position. Finally, the sensors were covered by hot similar material and it was smashed. The model test used a kind of self-healing material, so it did not affect the mechanical properties of the model test on the whole.

In order to observe the variation of temperature in the process, cold water (temperature of the water was $9.2^{\circ} \mathrm{C}$, while room temperature was $13.5^{\circ} \mathrm{C}$ ) was used in the experiment, and red ink was mixed in water to mark the position of the water inrush. FBG sensors work well and have no effect to others after embedded in the model test. They monitor the signals in real-time and provide a novel and effective approach for prewarning against water inrush in coalmine.

The experiment was carried out in twelve steps, and the first step was injecting water into the model. The process of water injection took about 32 minutes, and a constant temperature field, strain field, displacement field, and seepage field formed during the process, which was regarded as the first step. The second step was excavation of 


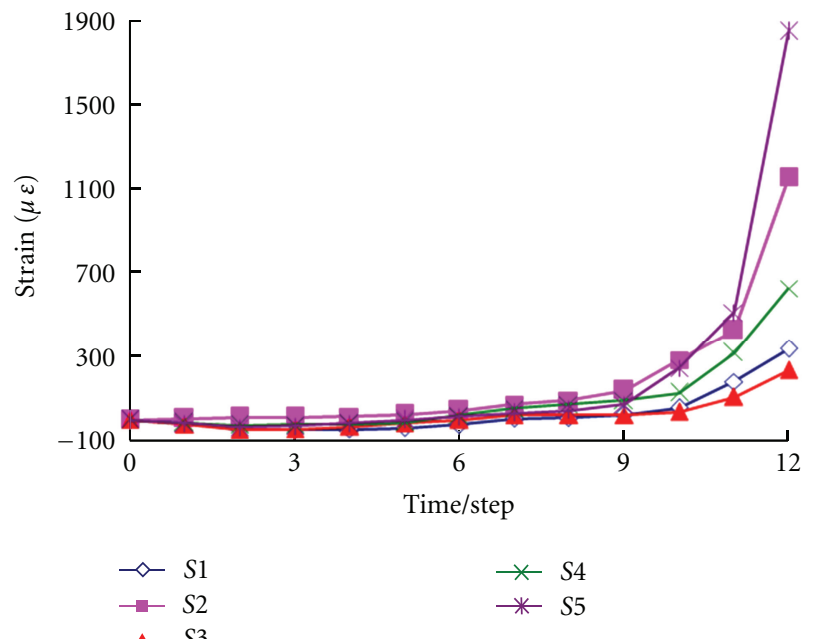

(a)

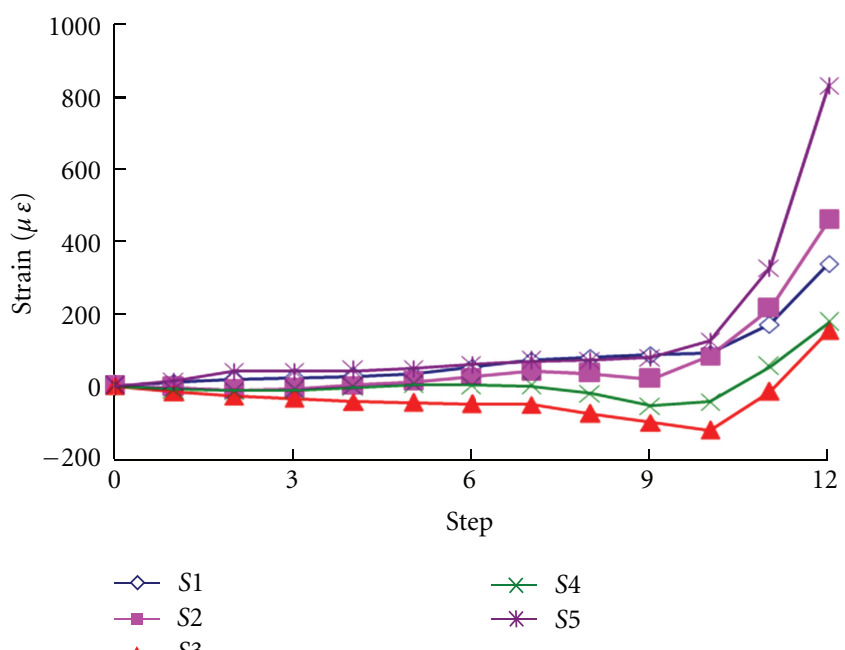

(b)

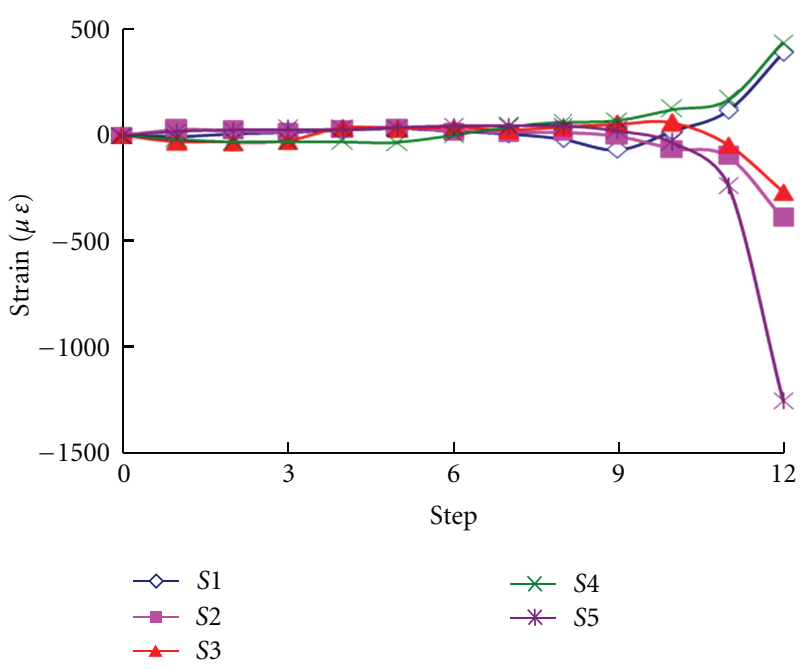

(c)

Figure 10: Curves of strain, (a) strain at $x$ direction, (b) strain at $z$ direction, and (c) shear strain.

the laneway consecutively, and there was water permeating from the roof of the laneway. Excavation of the mining area started from the third step to the tenth step. Manual excavating method was employed, and it was completed in a single operation. Excavation area expanded sideways $10 \mathrm{~cm}$ at a time. When excavation of the mining area was completed, water pressure still existed. After a period of time, information monitored by FBG sensors changed greatly, and the process was regarded as the eleventh step. Nevertheless, the duration of different kinds of FBG sensors was different. Eventually, the impermeable layer of the model collapsed suddenly, and it was the twelfth step.

Unfortunately, FBG strain sensor, FBG displacement sensor, and FBG seepage sensor at point 6 were all destroyed in the excavating process, while other FBG sensors registered information in real time. The results were shown in Figures $9,10,11$, and 12 , respectively.
4.2. Results of the Experiment. Temperature declined obviously under mining activity and under the effect of water pressure at the second step, in which the temperature at point 5 changed the most, and the amplitude reached up to $2.9^{\circ} \mathrm{C}$. Temperature at point 2 changed up to $2.2^{\circ} \mathrm{C}$, and temperature at other points changed about $2^{\circ} \mathrm{C}$ as shown in Figure 9. Moreover, in this step, temperature at point 5 changed firstly, and then temperature at point 3 changed secondly. Thereafter, the temperature field of the model test was basically stable. That is to say cracks running across point 5 , point 2 , and point 3 maybe generated.

As shown in Figure 10, the strain in the $x$ direction and shear strain were almost invariant until the ninth step. Along the $z$ direction, the strain of point 3 and point 4 was negative from the eighth step to the tenth step. In other words, the phenomenon of stress concentration occurred, while the strain of other points increased slowly. At the tenth step, the strain at point 2 and point 5 changed greatly in the $x$ 


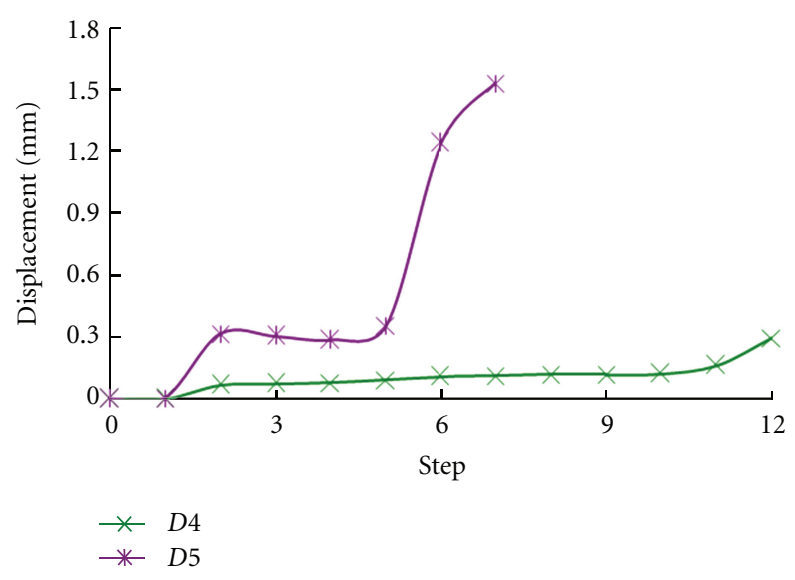

FIgURE 11: Curves of displacement.

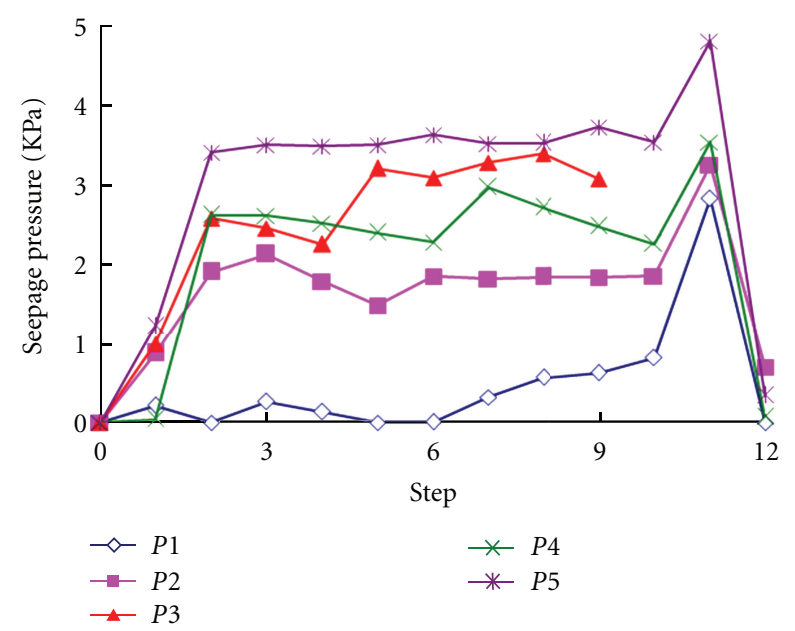

FIGURE 12: Curves of seepage pressure.

direction, while the strain at point 2 , point 5 , and point 3 changed greatly in the $z$ direction, and shear strain at point 2 and point 5 became negative under mining activity and water pressure, then partial rupture might occur, and seepage field would redistribute. At the eleventh step, strains in $x$ direction and $z$ direction were all increased greatly. Strain in $x$ direction exceeded $500 \mu \varepsilon$, and the variation rate was over $10 \mu \varepsilon$ per minute at point 5 . Nevertheless shear strain at point 5 changed up to $-200 \mu \varepsilon$, yet shear strain at point 1 and point 4 increased, while absolute value of negative shear strain at other points improved. Then it did yellow warning automatically. After 35 minutes, the collapsing of the impermeable layer resulted in variation of strain over a larger range.

In Figure 11, vertical displacement of the impermeable layer also changed slightly at the first step. But it changed up to $0.3 \mathrm{~mm}$ at the second step. The displacement at point 5 increased $0.92 \mathrm{~mm}$ at the sixth step, while the sensor was destroyed at the eighth step. Vertical displacement at point 4, which was compared with the numerical simulation result in [22], increases slowly in the process of mining, but it reached up to $0.164 \mathrm{~mm}$ at the eleventh step, and the system did

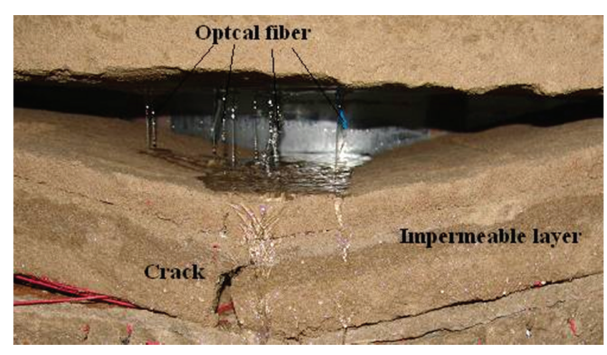

FIGURE 13: Collapse of impermeable layer.

orange warming. After 10 minutes, it increased $0.132 \mathrm{~mm}$, and water inrush occurred.

Figure 12 shows the variation of seepage pressure by step. The seepage pressure at point 2 , point 3 , and point 5 increased more than $0.8 \mathrm{KPa}$ at the first step, and then it changed greatly at the second step, among which the seepage pressure at point 5 was the largest. It is an indication that cracks in impermeable layer were generated. The seepage pressure was almost unaltered until the tenth step. The seepage pressure at point 3 also increased greatly in the excavation process, but unfortunately the sensor was destroyed at the tenth step. Seepage pressure all exceeded $2.8 \mathrm{KPa}$, and it did red alarm 8 seconds before the disaster happening, which was regarded as the eleventh step. It indicated that the cracks in the impermeable layer expanded. While at the twelfth step, seepage pressure all declined under $0.7 \mathrm{KPa}$, which demonstrated that the cracks in the impermeable layer expanded to point 1 and point 4 , while the cracks at point 5 run through the roof of the coalmine tunnel.

4.3. Fusion of the Signals. Temperature, strain, displacement, and seepage pressure signals were fused synthetically and the results were summarized as follows.

(1) The activity of injecting water to the model had implicit effects on temperature, strain, and displacement. But the temperature field, strain field, displacement field, and seepage field redistributed and balanced at a certain degree under normal mining activity.

(2) Strain exceeded the safety level (the safety level was set that the absolute value of strain exceeded $500 \mu \varepsilon$, and the variation rate was over $10 \mu \varepsilon$ per minute) 35 minutes before water inrush and after 25 minutes displacement at point 4 changed significantly, while temperature and seepage pressure did not change markedly at the same time. Moreover, strain, displacement, and seepage pressure all changed greatly when the impermeable layer collapsed and water inrush occurred. The FBG strain sensors obtained precursory information of water inrush first, and the FBG displacement sensor monitored it subsequently, while the FBG seepage pressure sensors described seepage pressure field imaginally. Therefore, the system could monitor the process of coalmine water inrush successfully, and abnormal variations in strain 
and displacement can be regarded as the portent of water inrush.

(3) It also indicated that cracks running across point 2, point 3, and point 5 generated the earliest, while cracks at point 1 and point 4 generated the last. In other words cracks at right generated earlier than those at left.

4.4. Visual Information. During the collapsing process, the fracture damage zones appeared first at the bottom of the impermeable layer due to mining activity and water pressure, and cracks at right generated earlier than those at left, which agreed with the conclusion from the monitoring system. When the cracks extended gradually to the aquifer and eventually to the water body, water inrush of the impermeable layer happened and the roof of the mining area collapsed ultimately as shown in Figure 13.

\section{Conclusion}

In order to avoid imperfectability, unreliability, and imprecision of a single parameter, a multiplexed FBG monitoring system is established. In this system FBG temperature sensors, FBG strain sensors, FBG displacement sensors, and FBG seepage pressure sensors which were specifically suitable for coalmine water inrush model test were developed. They had small volume and high resolution, and they could work well in coalmine water inrush monitoring. Then an intelligent diagnosis subsystem is employed, and the system can analyze signals synthetically and alarm automatically.

For the first time, the multiparameter FBG monitoring system was used in the coalmine water inrush model test, and it performed satisfactorily. The system exactly forecasts water inrush 35 minutes before the disaster. And one could conclude from the model test that high strain is a great threat to mine safety, and it might induce water inrush under certain conditions.

Moreover, the monitoring system can be employed in practical engineering with further improvement in the material of the strain block, the epoxy for the FBG strain sensors, the parameters of the spring in FBG displacement sensor, and the capability of the corrugated diaphragm in the FBG seepage pressure sensors. And other system improvements might be brought to bear by introducing wireless sensing technology to the system, so that it will become more convenient for practical application. On the other hand, since the system is relatively complicated to install, it is more suitable for long-term monitoring applications.

\section{Acknowledgments}

The authors acknowledge the support of the National Natural Science Foundation of Youth Science of China (41102183) and the National Natural Science Foundation of China (61074163).

\section{References}

[1] A. D. Kersey, M. A. Davis, H. J. Patrick et al., "Fiber grating sensors," Journal of Lightwave Technology, vol. 15, no. 8, pp. 1442-1463, 1997.

[2] S. Kim, S. Kim, J. Kwon, and B. Lee, "Fiber Bragg grating strain sensor demodulator using a chirped fiber grating," IEEE Photonics Technology Letters, vol. 13, no. 8, pp. 839-841, 2001.

[3] A. Iadicicco, A. Cusano, S. Campopiano, A. Cutolo, and M. Giordano, "Thinned fiber Bragg gratings as refractive index sensors," IEEE Sensors Journal, vol. 5, no. 6, pp. 1288-1295, 2005.

[4] Y. Zhan, S. Xue, Q. Yang, S. Xiang, H. He, and R. Zhu, "A novel fiber Bragg grating high-temperature sensor," Optik, vol. 119, no. 11, pp. 535-539, 2008.

[5] T. K. Gangopadhyay, M. Majumder, A. K. Chakraborty, A. K. Dikshit, and D. K. Bhattacharya, "Fibre Bragg grating strain sensor and study of its packaging material for use in critical analysis on steel structure," Sensors and Actuators A, vol. 150, no. 1, pp. 78-86, 2009.

[6] J. H. Ng, X. Zhou, X. Yang, and J. Hao, "A simple temperatureinsensitive fiber Bragg grating displacement sensor," Optics Communications, vol. 273, no. 2, pp. 398-401, 2007.

[7] T. Guo, Q. Zhao, H. Zhang et al., "Temperature-insensitive fiber Bragg grating dynamic pressure sensing system," Optics Letters, vol. 31, no. 15, pp. 2269-2271, 2006.

[8] L. J. Cashdollar and K. P. Chen, "Fiber Bragg grating flow sensors powered by in-fiber light," IEEE Sensors Journal, vol. 5, no. 6, pp. 1327-1331, 2005.

[9] J. Wang, Q. Sui, D. Feng, and M. Jiang, "Novel FBG vibration sensor based on matching filter demodulation," in Advanced Optical Manufacturing and Testing Technologies: Optical Test and Measurement Technology and Equipment, vol. 7283 of Proceedings of SPIE, pp. 251-256, May 2009.

[10] D. Song, Z. Wei, J. Zou, S. Yang, E. Du, and H.-L. Cui, "Pressure sensor based on fiber Bragg grating and carbon fiber ribbon-wound composite cylindrical shell," IEEE Sensors Journal, vol. 9, no. 7, pp. 828-831, 2009.

[11] P. Ferdinand, O. Ferragu, J. L. Lechien et al., "Mine operating accurate Stability control with optical fiber sensing and Bragg grating technology: the European BRITE/EURAM STABILOS project," Journal of Lightwave Technology, vol. 13, no. 7, pp. 1303-1313, 1995.

[12] A. Kerrouche, W. J. O. Boyle, T. Sun, and K. T. V. Grattan, "Design and in-the-field performance evaluation of compact FBG sensor system for structural health monitoring applications," Sensors and Actuators A, vol. 151, no. 2, pp. 107-112, 2009.

[13] C. Li, Y. G. Zhao, H. Liu, Z. Wan, C. Zhang, and N. Rong, "Monitoring second lining of tunnel with mounted fiber Bragg grating strain sensors," Automation in Construction, vol. 17, no. 5, pp. 641-644, 2008.

[14] H. Zhu, J. Yin, L. Zhang, J. Dong, K. Fung, and W. Jin, "Deformation monitoring of dam model test by optical fiber sensors," Chinese Journal of Rock Mechanics and Engineering, vol. 27, no. 6, pp. 1188-1194, 2008.

[15] H. Li, H. Sun, Y. Liu, X. Sun, and Y. Shang, "Application of optical fiber sensing technology to slope model test," Chinese Journal of Rock Mechanics and Engineering, vol. 27, no. 8, pp. 1703-1708, 2008.

[16] T. Yang, C. Tang, Z. Tan, W. Zhu, and Q. Feng, "State of the art of inrush models in rock mass failure and developing trend for prediction and forecast of groundwater inrush," Chinese Journal of Rock Mechanics and Engineering, vol. 26, no. 2, pp. 268-277, 2007. 
[17] D.-X. Cheng, Mechanical Design Handbook, Chemical Industry Press, Beijing, China, 2004.

[18] B. Lee and Y. Jeong, "Interrogation techniques for fiber grating sensors and the theory of fiber gratings," in Fiber Optic Sensors, F. T. S. Yu and S. Yin, Eds., pp. 295-382, Marcel Dekker, New York, NY, USA, 2002.

[19] J. Wang, D. Feng, Q. Sui, and B. Liu, "Study of optical fiber Bragg grating seepage pressure sensor based on draw-bar structure," Acta Optica Sinica, vol. 30, no. 3, pp. 686-691, 2010.

[20] H.-W. Fu, X.-G. Qiao, and J.-M. Fu, "Studying on the plat metal diaphragm based fiber Bragg gratings pressure sensing," Acta Photonica Sinica, vol. 33, no. 9, pp. 1119-1122, 2004.

[21] W. Jing, L. Shu-cai, S. Qing-mei et al., "Study of FBG strain sensors based on similar materiel in zonal disintegration model test[J]," Journal of China Coal Society, vol. 37, no. 9, pp. 1570-1575, 2012.

[22] L. I. Liping, Study on catastrophe evolution mechanism of karst water inrush and its engineering application of high risk karst tunnel [Ph.D. dissertation], Jinan, China, 2009. 

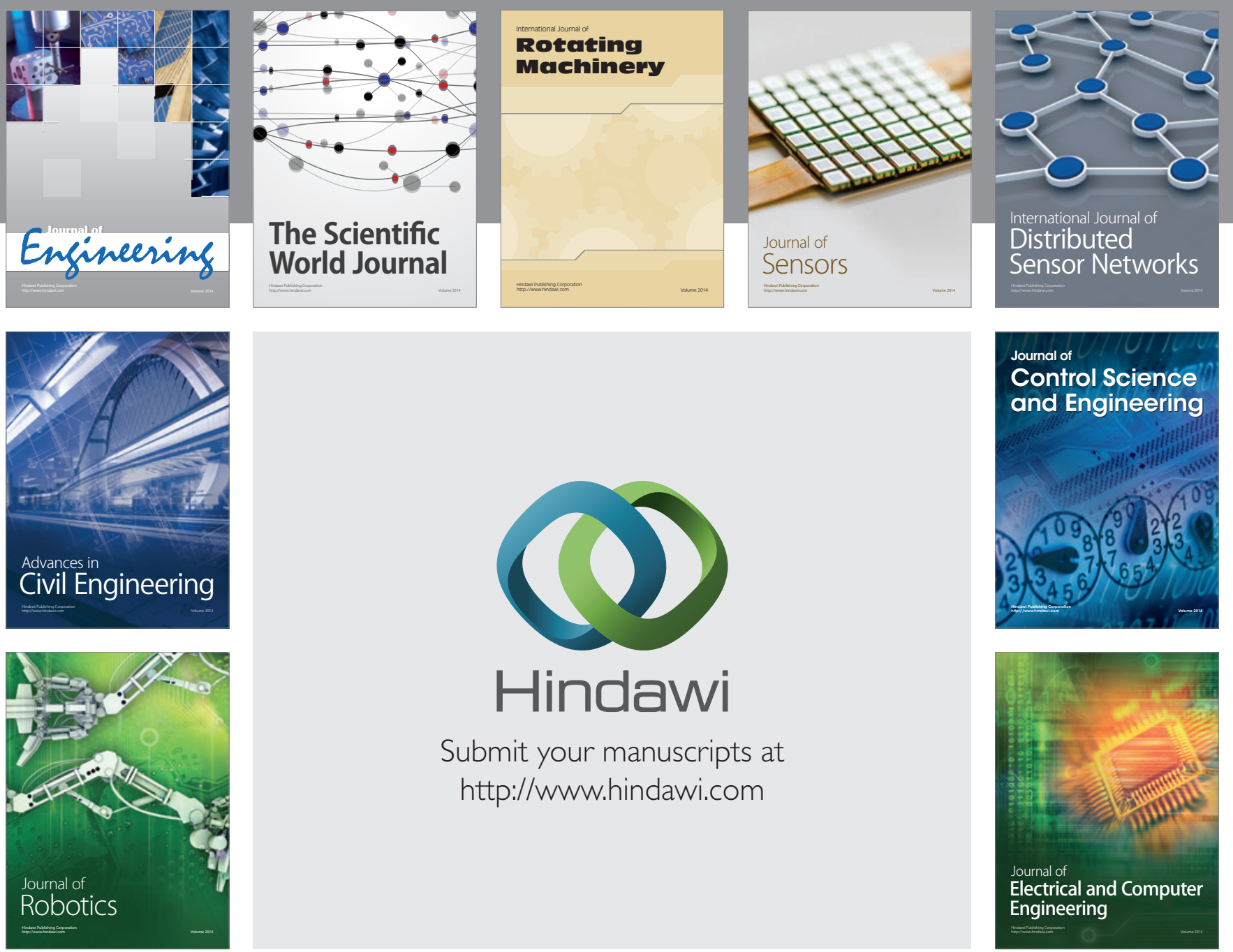

Submit your manuscripts at

http://www.hindawi.com
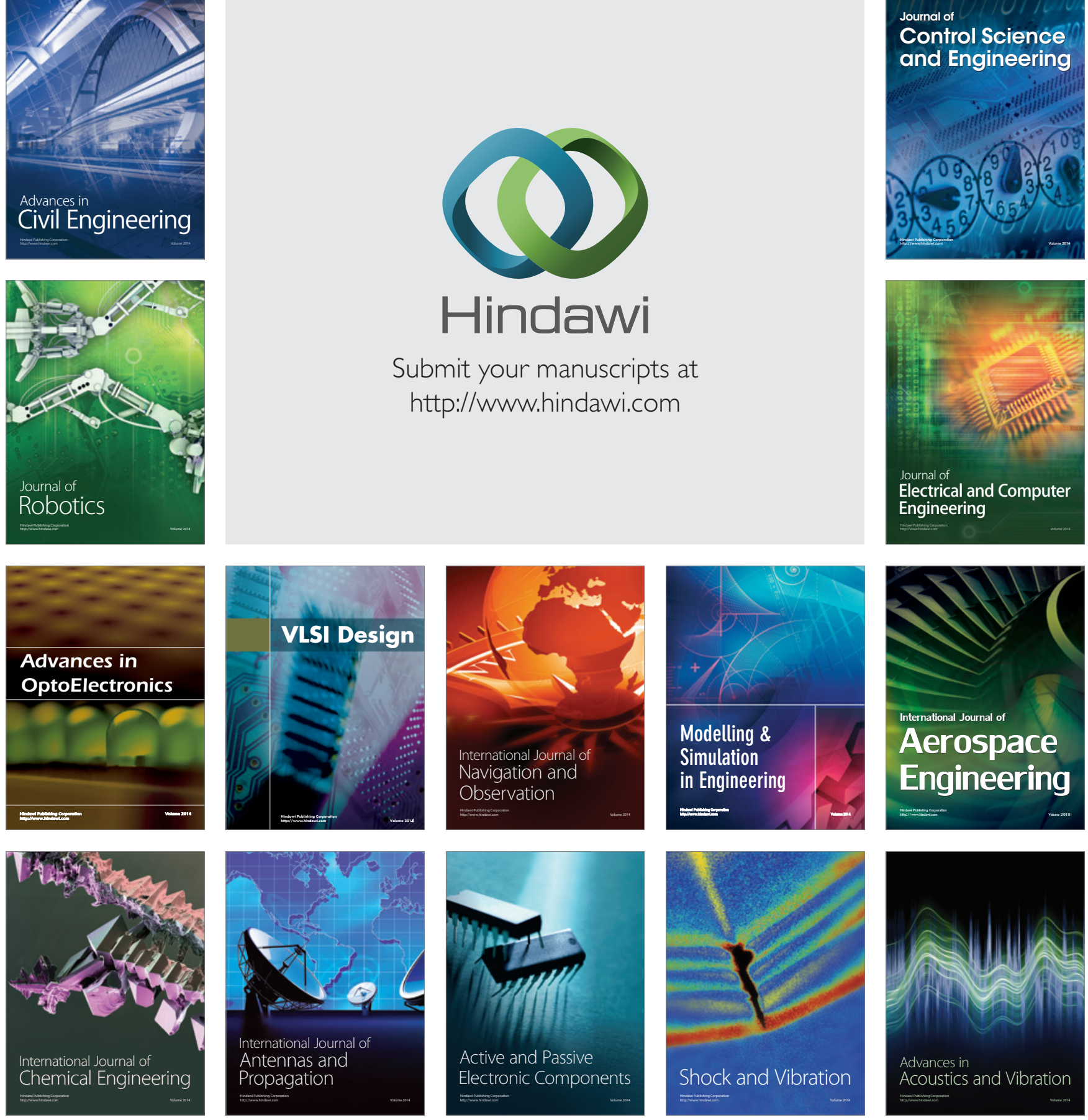